\title{
OCORRÊNCIA DE CONDIÇÕES AMBIENTAIS PARA A INDUÇÃO DO FLORESCIMENTO DE LARANJEIRAS NO ESTADO DE SÃO PAULO ${ }^{1}$
}

\author{
RAFAEL VASCONCELOS RIBEIRO ${ }^{2,3}$, EDUARDO CARUSO MACHADO², ORIVALDO BRUNINI ${ }^{2,3}$
}

\begin{abstract}
RESUMO - O presente estudo objetivou avaliar a ocorrência de condições ambientais propícias para a indução do florescimento de laranjeiras no Estado de São Paulo, considerando como fatores de indução a baixa temperatura, dada pelo número de horas de frio abaixo de $13^{\circ} \mathrm{C}(\mathrm{NHF})$, e a deficiência hídrica acumulada nos meses de junho, julho e agosto $\left(\mathrm{DEF}_{\mathrm{I}}\right)$, dada pelo balanço hídrico climatológico. Os cálculos e as estimativas foram realizados a partir dos dados de temperatura máxima e mínima diária e precipitação diária dos últimos 5 a 14 anos, dependendo da localidade. Foram consideradas áreas representativas das principais regiões produtoras de citros, onde os plantios estão em expansão ou áreas com potencial para exploração citrícola, sendo: Barretos, Bauru, Botucatu, Catanduva, Itapetininga, Itapeva, Jaboticabal, Jaú, Limeira, Lins, Matão, Mococa, Ourinhos, Piracicaba, São José do Rio Preto e Votuporanga. Com exceção de Botucatu, Itapetininga, Itapeva e Ourinhos, onde a indução do florescimento ocorre por baixa temperatura, a deficiência hídrica é o principal fator de indução nas demais regiões. Já nas regiões de Jaú, Limeira e Piracicaba, a indução do florescimento é ocasionada pelos dois fatores. A influência de NHF na indução do florescimento é mais variável se comparada à $\mathrm{DEF}_{\mathrm{I}}$. Em 1996, 2000 e 2004, NHF foi superior a 300 h na maioria das localidades estudadas, mesmo em áreas onde a baixa temperatura não é comum, ex. Barretos, S. J. Rio Preto e Votuporanga. Em relação à deficiência hídrica, a maioria das localidades apresentou esse tipo de influência ambiental no período analisado, sendo as menores ocorrências observadas em Itapeva e Itapetininga (54,5 e 72,7\% dos anos, respectivamente). Situação atípica ocorreu em 2004 , quando a deficiência hídrica variou de fraca $\left(10 \leq \mathrm{DEF}_{\mathrm{I}} \leq 30 \mathrm{~mm}\right)$ a moderada $(31 \leq \mathrm{DEF} \leq 69 \mathrm{~mm})$ na maioria das localidades. Concluindo, a deficiência hídrica é a principal variável ambiental durante o período de indução do florescimento de laranjeiras na região centro-norte do Estado de São Paulo, enquanto a baixa temperatura é predominante na região centro-sul do Estado, havendo influência desses dois fatores ambientais na região central.
\end{abstract}

Termos para indexação: Citrus, ecofisiologia, frio, seca.

\section{OCCURRENCE OF ENVIRONMENTAL CONDITIONS FOR FLOWERING INDUCTION OF SWEET ORANGE PLANTS IN THE STATE OF SÃO PAULO}

\begin{abstract}
This paper aimed to evaluate the occurrence of favorable environmental conditions for induction of sweet orange flowering in the State of São Paulo, considering as induction factors the low temperature, given by the number of chilling hours below $13^{\circ} \mathrm{C}(\mathrm{NCH})$, and the water deficiency accumulated during June, July and August $\left(\mathrm{DEF}_{\mathrm{w}}\right)$, given by the climatologic water balance. Calculations and estimatives were done using the maximum and minimum daily air temperature, as well as the daily rainfall recorded in the last 5 to 14 years, according to the local. There were considered areas in important regions of citrus production, where plantings are in expansion or in potential areas for citriculture, being: Barretos, Bauru, Botucatu, Catanduva, Itapetininga, Itapeva, Jaboticabal, Jaú, Limeira, Lins, Matão, Mococa, Ourinhos, Piracicaba, São José do Rio Preto and Votuporanga. Excepting Botucatu, Itapetininga, Itapeva and Ourinhos, where the flowering induction is triggered by low temperature, water deficiency is the principal factor for induction in other locals. At Jaú, Limeira and Piracicaba the flower induction is caused by both factors.

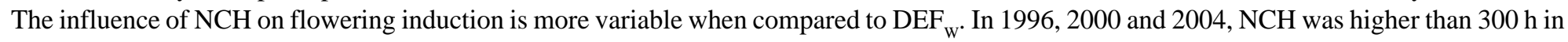
most of evaluated locals, even in areas where low temperature is uncommon, e.g. Barretos, S. J. Rio Preto and Votuporanga. In relation to the water deficiency, most of the regions showed this kind of environmental influence during the evaluated period, being lower occurrences observed in Itapeva and Itapetininga (54.5 and $72.7 \%$ of years, respectively). Unusual situation occurred in 2004, when water deficiency varied from weak $\left(10 \leq \mathrm{DEF}_{\mathrm{w}} \leq 30 \mathrm{~mm}\right)$ to moderate $\left(31 \leq \mathrm{DEF}_{\mathrm{w}} \leq 69 \mathrm{~mm}\right)$ in most of the locals. Concluding that $\mathrm{DEF}_{\mathrm{w}}$ is the main environmental variable during the period of flowering induction in sweet orange plants in the Central-North region of the State of São Paulo, while NCH is predominant in the Central-South region of the State, having influence of both environmental factors in the Central region.
\end{abstract}

Index terms: Citrus, cold, drought, ecophysiology.

\section{INTRODUÇÃO}

A produção de plantas cítricas é determinada primariamente pelo florescimento que, por sua vez, é condicionado pelo estado fisiológico das plantas (Goldschmidt et al., 1985; Garcia-Luis et al., 1988), assim como pelas condições ambientais (Ortolani et al., 1991; Chaikiattiyvos et al., 1994; Tubélis, 1995). Considerando-se o trinômio cultivo-clima-solo, o clima é o componente que influencia de sobremaneira no crescimento, produção e qualidade dos frutos (Reuther, 1977). Dentre as regiões produtoras de citros, as principais áreas estão situadas em clima subtropical úmido (entre $20^{\circ}$ e $40^{\circ}$ de latitude), onde a temperatura do ar e do solo atingem valores inferiores a $15^{\circ} \mathrm{C}$ no inverno, e a precipitação anual varia de 1.200 a 1.500 mm (Reuther, 1977; Davies, 1997). É importante considerar que essas condições climáticas são fatores estratégicos da citricultura brasileira, tornando-a altamente competitiva no cenário internacional (Ortolani et al., 1991).
Em relação à indução do florescimento em citros, sabe-se que baixas temperaturas e reduzida disponibilidade hídrica são os principais fatores ambientais que regulam tal processo (Southwick \& Davenport, 1986; Krajewski \& Rabe, 1995; Castro et al., 2001). Durante o inverno, as plantas diminuem o crescimento devido à baixa umidade do solo $\mathrm{e}$ temperatura $\left(<12-13^{\circ} \mathrm{C}\right)$, reduzindo o metabolismo e entrando em dormência (Reuther, 1977). Essa situação ambiental fornece estímulo para a transformação de gemas vegetativas em reprodutivas, sendo esse balanço possivelmente regulado quimicamente por intermédio de mudanças hormonais (Goldschmidt \& Monselise, 1970; Krajewski \& Rabe, 1995). Após as primeiras chuvas (>20 mm), o florescimento é iniciado e normalmente ocorre entre os meses de agosto e outubro no Estado de São Paulo (Tubélis, 1995), sendo resultado da indução ocorrida durante o pré-florescimento, isto é, 30 dias antes (Moss \& Muirhead, 1971). Quanto à importância dos fatores ambientais, alguns estudos têm sugerido que o papel da baixa temperatura é essencial para

\footnotetext{
(Trabalho 001-2006). Recebido: 01-01-2006. Aceito para publicação: 30-06-2006. Projeto realizado com apoio financeiro FEHIDRO.

${ }^{2}$ Eng. Agr., Dr. Pesquisador Científico, Centro de Pesquisa e Desenvolvimento em Ecofisiologia e Biofísica, Instituto Agronômico (IAC), Agência Paulista de Tecnologia dos Agronegócios (APTA), Secretaria de Agricultura e Abastecimento (SAA). Cx. Postal 28, 13001-970, Campinas-SP.E-mail: rafael@ iac.sp.gov.br, caruso@iac.sp.gov.br, brunini@iac.sp.gov.br.

${ }^{3}$ Centro Integrado de Informações Agrometeorológicas (CIIAGRO-IAC). Cx. Postal 28, 13001-970, Campinas-SP.
} 
o florescimento (Ben Mechlia \& Carroll, 1989; Aron et al., 1992), enquanto Chaikiattiyos et al. (1994) reportam florescimento reduzido em limões quando as plantas foram induzidas apenas pela baixa temperatura.

Devido à pressão biótica (pragas e doenças) e abiótica (temperaturas indesejáveis e baixa disponibilidade de água), há a tendência de expansão ou mesmo deslocamento das áreas produtoras para regiões com condições climáticas mais amenas e/ou onde exista reduzida pressão biótica. Assim sendo, o conhecimento das condições ambientais que propiciam o florescimento dos citros, uma das mais importantes fases fenológicas, é fundamental para o sucesso da citricultura nacional e manutenção do papel de destaque no comércio internacional. Nesse contexto, cabe ressaltar a participação expressiva do Estado de São Paulo, principal produtor mundial de citros (AGRIANUAL, 2005; IEA, 2005).

Este estudo objetivou avaliar a ocorrência de condições ambientais propícias para a indução do florescimento de citros no Estado de São Paulo, considerando como fatores indutivos o número de horas de frio e a deficiência hídrica.

\section{MATERIAL E MÉTODOS}

As regiões consideradas neste estudo são representativas das principais áreas produtoras de citros e/ou regiões onde o plantio tem se expandido (IEA, 2005) (Figura 1). Foram considerados os dados meteorológicos dos últimos anos, variando entre 14 (Piracicaba) e 5 (Ourinhos) anos, conforme a disponibilidade dos mesmos (Tabela 1).

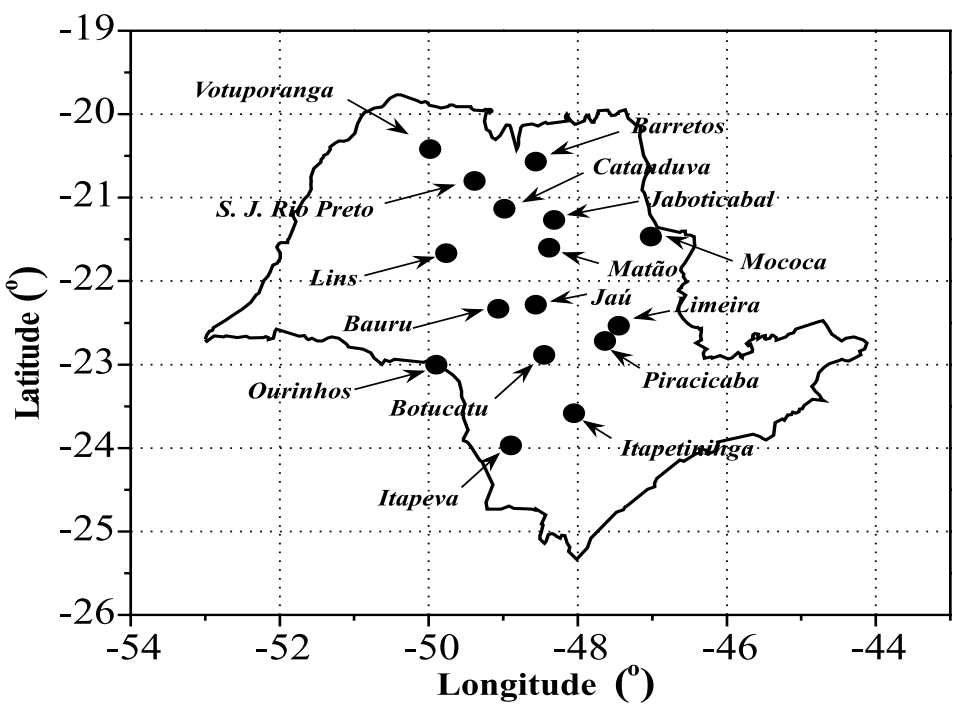

FIGURA 1 - Localização espacial das localidades avaliadas no Estado de São Paulo. Coordenadas geográficas e altitude são apresentadas na Tabela 1.
Os dados diários de temperatura do ar mínima e máxima, assim como os valores de precipitação foram obtidos da base de dados do Centro Integrado de Informações Agrometeorológicas (CIIAGRO, <ciiagro.iac.sp.gov.br>), Instituto Agronômico (IAC). A temperatura $\left({ }^{\circ} \mathrm{C}\right)$ média diária foi calculada a partir dos valores de temperatura máxima e mínima (termômetros de máxima e mínima). A temperatura média mensal foi calculada a partir dos valores de temperatura média diária. As temperaturas médias máxima e mínima de cada mês também foram calculadas. A precipitação mensal ( $\mathrm{mm}$ ) foi obtida pelo somatório dos valores de precipitação diária (pluviômetro convencional) durante o período considerado.

O número de horas de frio abaixo de $13^{\circ} \mathrm{C}(\mathrm{NHF})$ e a deficiência hídrica anual (DEF) e durante os meses de junho-julho-agosto ( $\mathrm{DEF}_{\mathrm{I}}$ ) foram estimados ano a ano para os períodos apresentados na Tabela 1. A estimativa de NHF foi realizada de acordo com a fórmula indicada por Pedro Jr. et al. (1979) para o Estado de São Paulo [NHF= 4482,8811 - $\left.\left(231,2112 \times \mathrm{T}_{\text {IULо }}\right)\right]$, considerando-se a temperatura média de julho $\left(\mathrm{T}_{\text {JULHO }}\right)$. Esse método de estimativa de NHF foi escolhido por ter sido desenvolvido para o Estado de São Paulo e por utilizar apenas $\mathrm{T}_{\text {JULHO }}$, haja vista que eram disponíveis apenas as temperaturas máximas e mínimas diárias na maioria das localidades. Os valores de DEF e DEF foram obtidos a partir de balanço hídrico climatológico, segundo Thornthwaite \& Mather (1955), utilizando planilha eletrônica desenvolvida por Rolim et al. (1998). No cálculo do balanço hídrico, foi considerada uma capacidade de água disponível (CAD) de 100 $\mathrm{mm}$, sendo esse valor o recomendado para cultivos perenes (Pereira et al., 2002).

As variáveis ambientais $\mathrm{NHF}$ e $\mathrm{DEF}_{\mathrm{I}}$ foram consideradas como indutoras do florescimento de laranjeiras. Embora NHF seja anual, sabe-se que os meses mais frios do ano ocorrem durante o inverno no Estado de São Paulo, sendo então, possivelmente, relacionados com as condições climáticas durante os meses de junho, julho e agosto. Considerando os valores médios máximos e mínimos

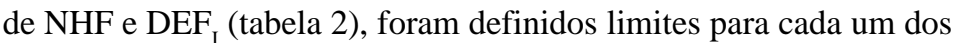
fatores de indução, indicando efeito fraco, médio ou forte. Valores de NHF entre 30 e $100 \mathrm{~h}$ sugerem fraca indução (+) por baixa temperatura, enquanto valores entre 101 e $299 \mathrm{~h}$ indicam efeito moderado (++) e valores superiores a $300 \mathrm{~h}$ um efeito forte (+++). Da mesma forma, foram estabelecidos limites para os valores de DEF quando considerada a influência da deficiência hídrica, ocorrendo indução fraca (+) quando $\mathrm{DEF}_{\mathrm{I}}$ varia entre 10 e $30 \mathrm{~mm}$, moderada entre 31 e 69 $\mathrm{mm}(++)$ e forte quando $\mathrm{DEF}_{\mathrm{I}}$ atinge valores superiores a $70 \mathrm{~mm}(+++)$.

\section{RESULTADOS E DISCUSSÃO}

De forma geral, as principais regiões produtoras de citros no

TABELA 1 - Localidades no Estado de São Paulo e períodos considerados no estudo.

\begin{tabular}{|c|c|c|c|}
\hline Localidade & Coordenada geográfica & Altitude (m) & Período* \\
\hline Barretos & $20^{\circ} 34^{\prime} \mathrm{S} ; 48^{\circ} 34^{\prime} \mathrm{O}$ & 518 & $1993-2005$ \\
\hline Bauru & $22^{\circ} 20^{\prime} \mathrm{S} ; 4^{\circ} 04^{\prime} \mathrm{O}$ & 615 & $1995-2005$ \\
\hline Botucatu & $22^{\circ} 53^{\prime} \mathrm{S} ; 48^{\circ} 27^{\prime} \mathrm{O}$ & 778 & $1995-2005$ \\
\hline Catanduva & $21^{\circ} 08^{\prime} \mathrm{S} ; 48^{\circ} 59^{\prime} \mathrm{O}$ & 488 & 1997-2005 \\
\hline Itapetininga & $23^{\circ} 35^{\prime} \mathrm{S} ; 4^{\circ} 03^{\prime} \mathrm{O}$ & 696 & $1995-2005$ \\
\hline Itapeva & $23^{\circ} 58^{\prime} \mathrm{S} ; 48^{\circ} 54^{\prime} \mathrm{O}$ & 649 & $1995-2005$ \\
\hline Jaboticabal & $21^{\circ} 16^{\prime} \mathrm{S} ; 48^{\circ} 19^{\prime} \mathrm{O}$ & 575 & $1993-2005$ \\
\hline Jaú & $22^{\circ} 17^{\prime} \mathrm{S} ; 48^{\circ} 34^{\prime} \mathrm{O}$ & 580 & 1993-2005 \\
\hline Limeira & $22^{\circ} 32^{\prime} \mathrm{S} ; 4^{\circ} 27^{\prime} \mathrm{O}$ & 639 & $1995-2005$ \\
\hline Lins & $21^{\circ} 40^{\prime} \mathrm{S} ; 4^{\circ} 46^{\prime} \mathrm{O}$ & 403 & $1995-2005$ \\
\hline Matão & $21^{\circ} 36^{\prime} \mathrm{S} ; 48^{\circ} 23^{\prime} \mathrm{O}$ & 551 & $1993-2005$ \\
\hline Mococa & $21^{\circ} 28^{\prime} \mathrm{S} ; 4^{\circ} 01^{\prime} \mathrm{O}$ & 665 & $1993-2005$ \\
\hline Ourinhos & $23^{\circ} 00^{\prime} \mathrm{S} ; 4^{\circ} 54^{\prime} \mathrm{O}$ & 466 & $2001-2005$ \\
\hline Piracicaba & $22^{\circ} 43^{\prime} \mathrm{S} ; 4^{\circ} 38^{\prime} \mathrm{O}$ & 580 & 1991-2005 \\
\hline São José do Rio Preto & $20^{\circ} 48^{\prime} \mathrm{S} ; 4^{\circ} 23^{\prime} \mathrm{O}$ & 468 & $1993-2005$ \\
\hline Votuporanga & $20^{\circ} 25^{\prime} \mathrm{S} ; 4^{\circ} 59^{\prime} \mathrm{O}$ & 505 & $1993-2005$ \\
\hline
\end{tabular}

*: 2005 não foi considerado nos cálculos de valores médios de temperatura e precipitação (Figura 1) e balanço hídrico (Figura 2). 
TABELA 2 - Valores médios* de temperatura do ar no mês de julho $\left(\mathrm{T}_{\mathrm{JULHO}}\right)$, número de horas de frio abaixo de $13^{\circ} \mathrm{C}(\mathrm{NHF})$, deficiência hídrica anual (DEF) e durante os meses de junho, julho e agosto $\left(\mathrm{DEF}_{\mathrm{I}}\right)$ e suas possíveis influências na indução do florescimento de laranjeiras.

\begin{tabular}{|c|c|c|c|c|c|c|}
\hline \multirow{2}{*}{ Localidade } & \multirow{2}{*}{$\mathrm{T}_{\text {JULHO }}\left({ }^{0} \mathrm{C}\right)$} & \multirow{2}{*}{ NHF (h) } & \multirow{2}{*}{ DEF (mm) } & \multirow{2}{*}{$\mathrm{DEF}_{\mathrm{I}}(\mathrm{mm})$} & \multicolumn{2}{|c|}{ Indução do florescimento** } \\
\hline & & & & & NHF & DEF $_{\text {I }}$ \\
\hline Barretos & 20,98 & 0,00 & 203,7 & 104,5 & & +++ \\
\hline Bauru & 20,10 & 0,00 & 82,1 & 52,4 & & ++ \\
\hline Botucatu & 18,00 & 321,30 & 10,4 & 8,7 & +++ & \\
\hline Catanduva & 21,00 & 0,00 & 236,8 & 108,8 & & +++ \\
\hline Itapetininga & 17,00 & 552,60 & 3,0 & 2,2 & +++ & \\
\hline Itapeva & 17,30 & 483,14 & 1,5 & 0,0 & +++ & \\
\hline Jaboticabal & 20,00 & 0,00 & 129,8 & 77,4 & & +++ \\
\hline Jaú & 19,10 & 66,98 & 29,8 & 23,3 & + & + \\
\hline Limeira & 18,20 & 275,06 & 33,7 & 24,7 & ++ & + \\
\hline Lins & 20,20 & 0,00 & 104,1 & 49,3 & & ++ \\
\hline Matão & 21,10 & 0,00 & 102,9 & 60,9 & & ++ \\
\hline Mococa & 19,80 & 0,00 & 87,0 & 55,8 & & ++ \\
\hline Ourinhos & 19,00 & 90,10 & 15,8 & 2,0 & + & \\
\hline Piracicaba & 18,00 & 321,30 & 20,2 & 17,3 & +++ & + \\
\hline São José do Rio Preto & 20,90 & 0,00 & 216,3 & 86,8 & & +++ \\
\hline Votuporanga & 21,00 & 0,00 & 163,3 & 82,9 & & +++ \\
\hline
\end{tabular}

*: Cálculos considerando o período indicado na Tabela 1;

$* *: \mathrm{NHF} \geq 300 \mathrm{~h}=$ efeito forte $(+++) ; 101 \leq \mathrm{NHF} \leq 299 \mathrm{~h}=$ efeito moderado $(++) ; 30 \leq \mathrm{NHF} \leq 100 \mathrm{~h}=$ efeito fraco $(+)$;

$\mathrm{DEF}_{\mathrm{I}} \geq 70 \mathrm{~mm}=$ efeito forte $(+++) ; 31 \leq \mathrm{DEF}_{\mathrm{I}} \leq 69 \mathrm{~mm}=$ efeito moderado $(++) ; 10 \leq \mathrm{DEF}_{\mathrm{I}} \leq 30 \mathrm{~mm}=$ efeito fraco $(+)$.

Estado de São Paulo apresentam um período úmido e quente, compreendendo os meses de novembro a fevereiro (verão), e outra época com baixos índices pluviométricos e menor temperatura, abrangendo os meses de junho, julho e agosto (inverno) (Figura 2). Segundo Ortolani et al. (1991), o clima tropical de atitude ou mesotérmico úmido predomina em cerca de $90 \%$ do território do Estado de São Paulo. As temperaturas médias mensais máximas variam entre 22 e $34^{\circ} \mathrm{C}$, ao passo que as mínimas variam entre 10 e $21^{\circ} \mathrm{C}$ (Figura 2 ). Já os totais médios de chuva anual variam entre 1.199 (Catanduva) e $1.588 \mathrm{~mm}$ (Itapeva). Essas condições ambientais estão dentro de uma faixa adequada para o crescimento e desenvolvimento de citros, quando consideradas as diferentes fases fenológicas que compreendem desde o florescimento e frutificação até a dormência das plantas nos meses mais frios (Reuther, 1977; Davies \& Albrigo, 1994). Tubélis (1995) ainda cita que o sucesso da citricultura paulista é baseado na qualidade física dos solos, que permitem boa drenagem e bom desenvolvimento radicular, aliado ao fato de que a ocorrência de geadas fracas é baixa, e a região não está sujeita a períodos de baixa carga energética (insolação).

Entretanto, quando comparadas as regiões produtoras de citros no Estado de São Paulo, observou-se a ocorrência de condições ambientais bem distintas em relação aos regimes de temperatura do ar e precipitação (Figura 2). As áreas mais ao Norte do Estado têm como característica temperatura do ar mais elevada e menor ocorrência de chuvas durante o inverno. Representantes dessa região, as localidades de Barretos, Catanduva, São José do Rio Preto e Votuporanga apresentam temperaturas médias ao redor de $27^{\circ} \mathrm{C}$ nos meses mais quentes do ano e valores de precipitação no mês mais seco inferiores a $15 \mathrm{~mm}$ (Figura 2A,D,O,P). Segundo o zoneamento agrícola da citricultura para o Estado de São Paulo (Camargo et al., 1974), essas localidades estão compreendidas na zona Z2, caracterizada por ser apta para o cultivo dos citros com restrições por apresentarem deficiência hídrica acentuada. O zoneamento também agrupa na zona Z2 as localidades de Jaboticabal, Piracicaba, Jaú e Lins (Camargo et al., 1974). Entretanto, essas áreas têm apresentado condições ambientais nesses últimos anos mais próximas das características climáticas da zona Z1, a que pertencem as localidades de Limeira, Botucatu e Bauru. Essa zona é apta para o cultivo de citros sem restrições térmicas e hídricas. Já as áreas mais frias, representadas por Itapeva e Itapetininga, pertencem à zona Z3, considerada marginal e inapta por não apresentar estação seca.
Quando considerados os balanços hídricos médios dos últimos anos (Figura 3), notam-se três grupos de localidades segundo os valores de deficiência hídrica anual: com deficiência hídrica acentuada (Barretos, Catanduva, São José do Rio Preto e Votuporanga); com deficiência hídrica moderada (Bauru, Jaboticabal, Lins, Matão e Mococa); e com deficiência hídrica pequena ou inexistente (Itapetininga, Itapeva, Jaú, Limeira, Ourinhos, Piracicaba e Botucatu). Assim, algumas localidades das zonas Z2 e Z1 não apresentaram deficiência hídrica acentuada (Figura 3), sendo observados valores máximos de DEF ao redor de $20 \mathrm{~mm}$ em Jaú (Figura 3H), $10 \mathrm{~mm}$ em Limeira (Figura 3I), <10 mm em Ourinhos (Figura 3M) e inferiores a $15 \mathrm{~mm}$ em Botucatu e Piracicaba (Figura 3C,N).

Uma vez que as condições climáticas dos últimos anos nas principais regiões citrícolas foram caracterizadas, o próximo passo é a identificação de quais fatores ambientais estão envolvidos na indução do florescimento de laranjeiras em cada localidade. De acordo com os dados médios de $\mathrm{DEF}_{\mathrm{I}}$, a deficiência hídrica é o principal indutor do florescimento em quase todas as localidades estudadas, com exceção de Botucatu, Itapetininga, Itapeva e Ourinhos (Tabela 2). Em Ourinhos e considerando apenas a condição ambiental média do período avaliado (2001-2005), há um fraco efeito da baixa temperatura (Tabelas 1 e 2). Existem ainda regiões em que há a influência de ambas as variáveis ( $\mathrm{NHF}$ e DEF ), tais como Limeira, Jaú e Piracicaba (Tabela 2), onde possivelmente ocorre um efeito aditivo entre esses estresses ambientais no florescimento de laranjeiras. Considerando-se a ocorrência dos dois fatores de indução, o efeito da baixa temperatura varia de fraco (Jaú) a moderado (Limeira) quando ocorre em conjunto com a deficiência hídrica, com exceção de Piracicaba (Tabela 2). Entretanto, faz-se necessário avaliar a variação dos fatores de indução ano a ano para melhor visualização da ocorrência de condições ambientais propícias para a indução do florescimento e suas possíveis consequiências para a produção, assim como para detectar a ocorrência de anos atípicos para a citricultura, quando considerada a indução do florescimento.

Em relação à influência da baixa temperatura na indução do florescimento (Tabela 3), pode-se notar que o ano de 1996 foi especialmente frio, quando houve efeito de NHF mesmo em Barretos (efeito moderado), onde, segundo os dados meteorológicos médios, a indução ocorre preferencialmente pela deficiência hídrica (efeito forte). Os anos de 2000 e 2004 também foram frios, porém em menor intensidade, promovendo efeito de moderado a forte em relação à 

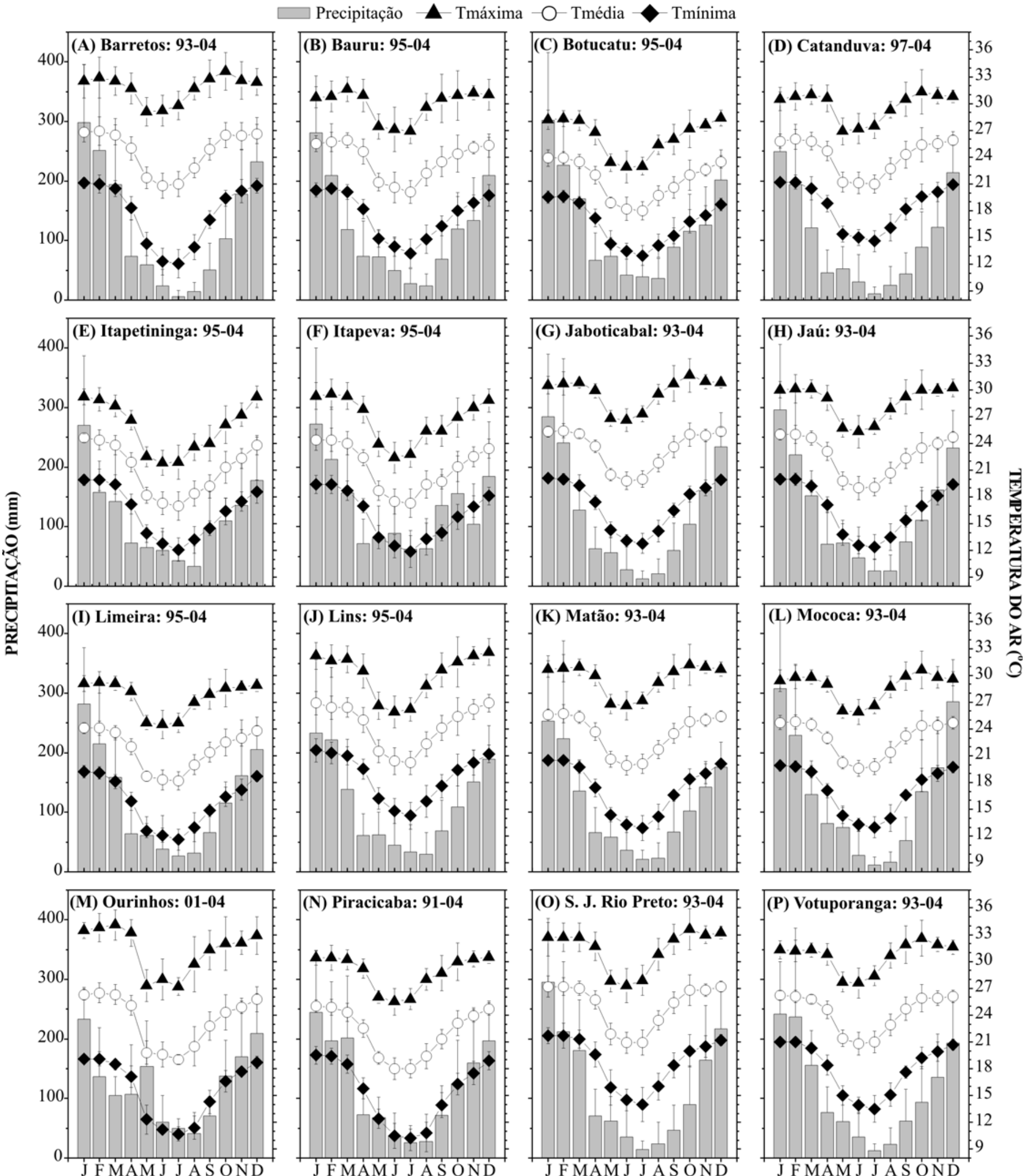

\section{MESES}

FIGURA 2 - Temperatura mensal máxima, mínima e média e precipitação mensal em localidades do Estado de São Paulo. Os dados se referem aos valores médios durante o período indicado na Tabela 1 . Cada ponto e barra representam o valor médio mensal \pm desvio-padrão.

indução do florescimento (Tabela 3) nas regiões onde esse fator é predominante (Tabela 2). Assim, o efeito da baixa temperatura, seja fraco, moderado ou forte, ocorreu em pelo menos $80 \%$ dos anos em Botucatu, Itapetininga, Itapeva, Limeira e Piracicaba. Em Ourinhos, a baixa temperatura durante o período de indução do florescimento ocorreu em $60 \%$ dos anos, enquanto em Jaboticabal, Jaú e Mococa essa condição ambiental aconteceu entre 23 e 38,5\% dos anos. Já em localidades onde o principal fator de indução é a deficiência hídrica (Tabela 2), a baixa temperatura ocorreu em apenas $18 \%$ dos anos em
Lins, 15,4\% em Matão e Votuporanga, e em menos que $10 \%$ dos anos em Barretos e São José do Rio Preto, chegando a não ocorrer em Catanduva durante o período estudado (1997-2005).

A deficiência hídrica do solo durante o período de indução do florescimento ocorreu em 100\% dos anos em Barretos, Bauru, Catanduva, Jaboticabal, Limeira, Lins, Mococa, Piracicaba, São José do Rio Preto e Votuporanga, onde o efeito variou de moderado a forte (Tabela 3). Já nas regiões de Botucatu, Jaú, Matão e Ourinhos, a ocorrência foi igual ou superior a $80 \%$, sendo observados valores ao 

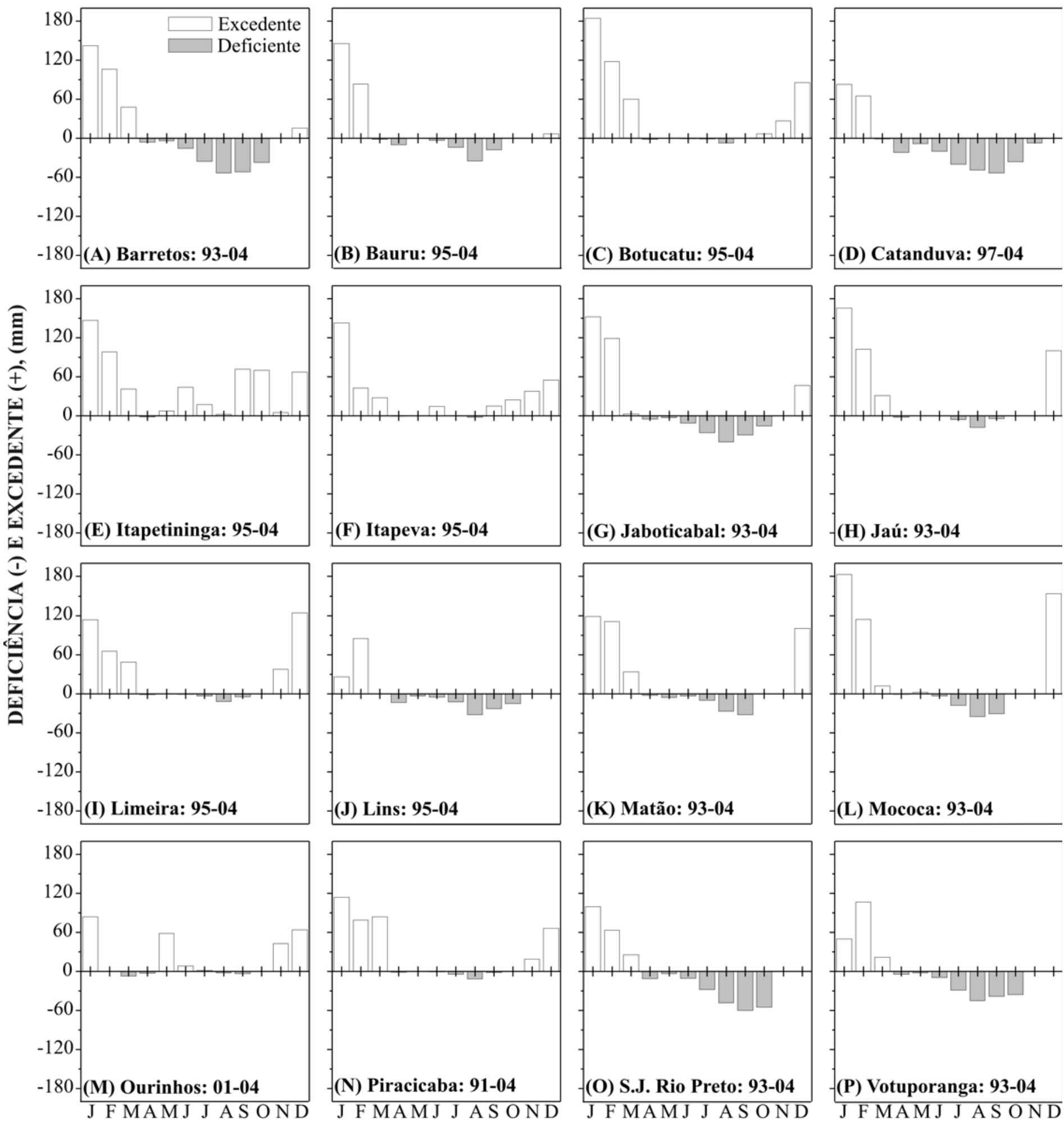

MESES

FIGURA 3 - Extrato simplificado do balanço hídrico climatológico (Thornthwaite \& Mather, 1955) médio em localidades do Estado de São Paulo. Os dados se referem aos valores médios do período indicado na Tabela 1. Valores positivos e negativos indicam excesso e deficiência, respectivamente. $\mathrm{CAD}=100 \mathrm{~mm}$.

redor de $73 \%$ em Itapetininga e 55\% em Itapeva. Nessas últimas duas localidades, o efeito variou de fraco a moderado, com exceção do ano de 2005 (Tabela 3) em que a deficiência hídrica durante o período de indução (junho-julho-agosto), na grande maioria das localidades estudadas, variou de moderada a forte $\left(\mathrm{DEF}_{\mathrm{I}}>30 \mathrm{~mm}\right)$. Um ponto que chama a atenção é a deficiência hídrica de 2004 (Tabela 3), quando as únicas localidades avaliadas a apresentarem forte influência de $\mathrm{DEF}_{\mathrm{I}}$ foram Bauru e Votuporanga.

Um aspecto importante a considerar é que o efeito ambiental na indução do florescimento em determinado ano resultará em alterações na produção do ano subseqüente, haja vista que a maioria das variedades cultivadas apresenta intervalo entre colheitas superior a oito meses. Ou seja, se a fraca indução do florescimento pela seca ocorrida em 2004 resultou em menor florada nas regiões onde a deficiência hídrica é o principal fator de indução, ex. Barretos, Bauru, Catanduva, Jaboticabal e São José do Rio Preto, dentre outros (Tabela 2), as conseqüências na produção de citros serão verificadas na colheita de 2005 (ano agrícola 2004-2005). Mesmo em localidades onde ambos os fatores de indução são atuantes, ex. Limeira, esperase alguma alteração na quantidade de flores formadas, desde que os efeitos possam ser aditivos. Em relação a esse tópico, alguns estudos têm demonstrado papel predominante da seca (Chaikiattiyos et al., 1994), do frio (Ben Mechlia \& Carroll, 1989; Valiente \& Albrigo, 2004) ou de ambos (Southwick \& Davenport, 1986; Krajewski \& Rabe, 1995) 
TABELA 3 - Ocorrência de condições ambientais para a indução do florescimento de laranjeiras, considerando como fatores de indução (FI) o número de horas de frio abaixo de $13^{\circ} \mathrm{C}(\mathrm{NHF})^{*}$ e deficiência hídrica acumulada durante junho, julho e agosto $\left(\mathrm{DEF}_{\mathrm{I}}\right)^{* *}$

\begin{tabular}{|c|c|c|c|c|c|c|c|c|c|c|c|c|c|c|c|c|c|}
\hline \multirow{2}{*}{ Localidade } & \multirow{2}{*}{ FI } & \multicolumn{15}{|c|}{ Ano } & \multirow{2}{*}{$\begin{array}{c}\text { Ocorrência } \\
(\%)\end{array}$} \\
\hline & & 1991 & 1992 & 1993 & 1994 & 1995 & 1996 & 1997 & 1998 & 1999 & 2000 & 2001 & 2002 & 2003 & 2004 & 2005 & \\
\hline \multirow{2}{*}{ Barretos } & NHF & ----- & ----- & & & & ++ & & & & & & & & & & 7,7 \\
\hline & $\mathrm{DEF}_{\mathrm{I}}$ & ----- & ----- & +++ & +++ & +++ & ++ & ++ & +++ & +++ & +++ & +++ & +++ & +++ & ++ & +++ & 100,0 \\
\hline \multirow{2}{*}{ Bauru } & NHF & ----- & ----- & ----- & ----- & & ++ & & & & +++ & & & & ++ & & 27,3 \\
\hline & $\mathrm{DEF}_{\mathrm{I}}$ & ----- & ----- & ----- & ----- & +++ & ++ & ++ & ++ & ++ & ++ & ++ & +++ & +++ & +++ & +++ & 100,0 \\
\hline \multirow{2}{*}{ Botucatu } & NHF & ----- & ----- & ----- & ----- & & +++ & ++ & ++ & ++ & +++ & ++ & +++ & ++ & +++ & +++ & 90,9 \\
\hline & $\mathrm{DEF}_{I}$ & ----- & ----- & ----- & ----- & + & ++ & ++ & + & ++ & ++ & & + & + & + & ++ & 90,9 \\
\hline \multirow{2}{*}{ Catanduva } & NHF & ----- & ----- & ----- & ----- & ----- & ----- & & & & & & & & & & 0,0 \\
\hline & $\mathrm{DEF}_{\mathrm{I}}$ & ----- & ----- & ----- & ----- & ----- & ----- & ++ & ++ & +++ & +++ & +++ & +++ & +++ & ++ & +++ & 100,0 \\
\hline \multirow{2}{*}{ Itapetininga } & NHF & ----- & ----- & ----- & ----- & ++ & +++ & +++ & +++ & & +++ & +++ & +++ & +++ & +++ & +++ & 90,9 \\
\hline & $\mathrm{DEF}_{\mathrm{I}}$ & ----- & ----- & ----- & ----- & + & + & + & & + & ++ & & + & & + & +++ & 72,7 \\
\hline \multirow{2}{*}{ Itapeva } & NHF & ----- & ----- & ----- & ----- & & +++ & & ++ & +++ & +++ & +++ & +++ & ++ & +++ & +++ & 81,8 \\
\hline & $\mathrm{DEF}_{\mathrm{I}}$ & ---- & ---- & ---- & ----- & & + & & & + & + & & + & & + & ++ & 54,5 \\
\hline \multirow{2}{*}{ Jaboticabal } & NHF & ----- & ----- & & & & ++ & & & & ++ & & & & + & + & 30,8 \\
\hline & $\mathrm{DEF}_{\mathrm{I}}$ & ----- & ----- & ++ & +++ & +++ & +++ & ++ & ++ & +++ & ++ & +++ & +++ & ++ & ++ & + & 100,0 \\
\hline \multirow{2}{*}{ Jaú } & NHF & ----- & ----- & & & & +++ & & & & +++ & & ++ & & ++ & ++ & 38,5 \\
\hline & $\mathrm{DEF}_{\mathrm{I}}$ & ----- & ----- & & +++ & +++ & +++ & + & + & ++ & ++ & ++ & ++ & ++ & + & +++ & 92,3 \\
\hline \multirow{2}{*}{ Limeira } & NHF & ----- & ----- & ----- & ----- & & +++ & ++ & ++ & ++ & +++ & + & +++ & + & +++ & ++ & 90,9 \\
\hline & $\mathrm{DEF}_{\mathrm{I}}$ & ----- & ----- & ----- & ----- & +++ & ++ & + & ++ & +++ & ++ & ++ & ++ & +++ & + & +++ & 100,0 \\
\hline \multirow{2}{*}{ Lins } & NHF & ----- & ----- & ----- & ----- & & +++ & & & & & & & & ++ & & 18,2 \\
\hline & $\mathrm{DEF}_{\mathrm{I}}$ & ----- & ----- & ---- & ----- & +++ & +++ & ++ & +++ & +++ & ++ & + & +++ & +++ & + & +++ & 100,0 \\
\hline \multirow{2}{*}{ Matão } & NHF & ----- & ----- & & & & ++ & & & & ++ & & & & & & 15,4 \\
\hline & $\mathrm{DEF}_{\mathrm{I}}$ & ----- & ----- & & ++ & +++ & +++ & ++ & +++ & +++ & +++ & +++ & +++ & +++ & + & +++ & 92,3 \\
\hline \multirow{2}{*}{ Mococa } & NHF & ----- & ----- & & & & ++ & & & & ++ & & & & ++ & & 23,1 \\
\hline & $\mathrm{DEF}_{\mathrm{I}}$ & ----- & ----- & + & ++ & +++ & ++ & ++ & +++ & +++ & +++ & +++ & +++ & +++ & + & +++ & 100,0 \\
\hline \multirow{2}{*}{ Ourinhos } & NHF & ---- & ----- & ---- & ----- & ----- & ----- & ---- & ----- & ----- & ----- & & ++ & & ++ & ++ & 60,0 \\
\hline & $\mathrm{DEF}_{\mathrm{I}}$ & ----- & ----- & ----- & ----- & ----- & ----- & ----- & ----- & ----- & ----- & + & + & & + & +++ & 80,0 \\
\hline \multirow{2}{*}{ Piracicaba } & NHF & +++ & ++ & ++ & +++ & + & +++ & + & ++ & ++ & +++ & + & +++ & ++ & +++ & ++ & 100,0 \\
\hline & $\mathrm{DEF}_{\mathrm{I}}$ & ++ & ++ & + & + & + & +++ & + & + & ++ & ++ & ++ & ++ & +++ & + & +++ & 100,0 \\
\hline \multirow{2}{*}{ S.J. Rio Preto } & NHF & ----- & ----- & ----- & & & & & & & ++ & & & & & & 8,3 \\
\hline & $\mathrm{DEF}_{\mathrm{I}}$ & ----- & ----- & ----- & +++ & +++ & +++ & ++ & ++ & +++ & +++ & +++ & +++ & ++ & ++ & +++ & 100,0 \\
\hline \multirow{2}{*}{ Votuporanga } & NHF & ----- & ----- & & & & + & & & & ++ & & & & & & 15,4 \\
\hline & $\mathrm{DEF}_{\mathrm{I}}$ & ----- & ----- & ++ & +++ & +++ & +++ & ++ & ++ & +++ & +++ & +++ & +++ & +++ & +++ & +++ & 100,0 \\
\hline
\end{tabular}

*: $\mathrm{NHF}>300 \mathrm{~h}=$ efeito forte $(+++) ; 101<\mathrm{NHF}<299 \mathrm{~h}=$ efeito moderado $(++) ; 30<\mathrm{NHF}<100 \mathrm{~h}=$ efeito fraco $(+)$;

$* *: \mathrm{DEF}_{\mathrm{I}} \geq 70 \mathrm{~mm}=$ efeito forte $(+++) ; 31 \leq \mathrm{DEF}_{\mathrm{I}} \leq 69 \mathrm{~mm}=$ efeito moderado $(++) ; 10 \leq \mathrm{DEF}_{\mathrm{I}} \leq 30 \mathrm{~mm}=$ efeito fraco $(+)$.

Ausência de dados é indicada por $\longrightarrow$.

no florescimento de citros, dependendo da região climática, isto é, subtropical ou tropical (Davies \& Albrigo, 1994). Em Limeira, o número total de estruturas reprodutivas foi superior em plantas submetidas à baixa temperatura e à deficiência hídrica quando comparadas às plantas induzidas apenas pelo frio (Ribeiro et al., dados não publicados).

Outro aspecto cuja atenção deve ser direcionada, referese ao número de flores e quantidade de frutos colhidos. Sabe-se que o pegamento de flores em plantas cítricas é baixo e varia entre 0,2 e 2,5\% (Erickson \& Brannaman, 1960; Bustan \& Goldschmidt, 1998), sendo esse valor função não apenas das condições ambientais (temperatura, vento, umidade do ar e solo), mas também do estado fisiológico das plantas, isto é, reservas nos tecidos para suportar o dreno exercido pelo crescimento reprodutivo e vegetativo, nutrição mineral e balanço hormonal, e da carga pendente de frutos (Reuther, 1977; Monselise \& Goren, 1978; Goldschmidt \& Golomb, 1982; Goldschmidt et al., 1985; Davies \& Albrigo, 1994). Esse fato sugere que uma grande quantidade de estruturas florais não necessariamente implica aumento da produção, representando um mecanismo evolutivo para a perpetuação da espécie. Períodos de estiagem acompanhados ou não por alta temperatura do ar durante e logo após o florescimento também reduzem o pegamento dos frutos, causando prejuízos à citricultura (Reuther, 1977; Davies \& Albrigo, 1994). Logo, é importante considerar que a indução adequada do florescimento é fundamental, mas não implica alta produção, visto que outros fatores (ambientais e endógenos) também concorrem para este processo.

\section{CONCLUSÕES}

A deficiência hídrica é a principal variável ambiental durante o período de indução do florescimento de laranjeiras na região centronorte do Estado de São Paulo, enquanto a baixa temperatura é predominante na região centro-sul. Esses dois fatores ambientais ocorrem em conjunto na região central do Estado de São Paulo, sendo ambas as variáveis possivelmente relacionadas à indução do florescimento de laranjeiras nessa área.

\section{REFERÊNCIAS}

AGRIANUAL 2005: anuário da agricultura brasileira. 10. ed. São Paulo: FNP Consultoria \& Comércio, 2004. 520p.

ARON, R.H.; GAT, Z.; LEE, C.M-S.; ERNER, Y. The distribution of the most favorable temperatures for citrus flower induction in Israel. International Journal of Biometeorology, New York, v.36, n.2, p.108-112, 1992.

BEN MECHLIA, N.; CARROLL, J.J. Agroclimatic modeling for the stimulation of phenology, yield and quality of crop production. I. Citrus response formulation. International Journal of Biometeorology, New York, v.33, n.1, p.36-51, 1989.

BUSTAN, A.; GOLDSCHMIDT, E.E. Estimating the cost of flowering in a grapefruit tree. Plant, Cell and Environment, Oxford, v.21, n.2, p.217-224, 1998.

CAMARGO, A.P.; PINTO, H.S.; PEDRO Jr., M.J.; BRUNINI, O.; ALFONSI, R.R.; ORTOLANI, A.A. Aptidão climática de culturas agrícolas. In: SECRETARIADEAGRICULTURADE SÃOPAULO. Zoneamento agrícola do Estado de São Paulo. São Paulo: CATI, 1974. p.109-149.

CASTRO, P.R.C.; MARINHO, C.S.; PAIVA, R.; MENEGUCCI, J.L.P. Fisiologia da produção dos citros. Informe Agropecuário, Belo Horizonte, v.22, n.209, p.26-38, 2001.

CHAIKIATTIYOS, S.; MENZEL, C.M.; RASMUSSEN, T.S. Floral induction in tropical fruit trees: effects of temperature and water supply. Journal of Horticultural Science, Kent, v.69, n.3, p.397415, 1994.

DAVIES, F.S. An overview of climatic effects on citrus flowering and fruit quality in various parts of the world. In: CITRUS FLOWERING \& FRUITING SHORT COURSE, 1., 1997, Lake Alfred. 
Proceedings... Lake Alfred: CREC/IFAS, 1997. Disponível em:

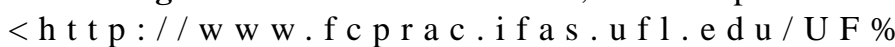
20 IFAS $\% 20 \mathrm{Sh}$ ort $\% 20 \mathrm{Course} \% 20 \mathrm{Proce}$ dings $/$ citrusflowering.htm>. Acesso em: 23 nov. 2005.

DAVIES, F.S.; ALBRIGO, L.G. Citrus. Wallingford: CAB International, 1994.254p.

ERICKSON, L.C.; BRANNAMAN, B.L. Abscission of reproductive structures and leaves of orange trees. Proceedings of the American Society for Horticultural Science, Geneva, v.75, p.222229, 1960.

GARCIA-LUIS, A.; FORNES, F.; SANZ, A.; GUARDIOLA, J.L. The regulation of flowering and fruit set in Citrus: relationship with carbohydrate levels. Israel Journal of Botany, Jerusalem, v.37, n.2-4, p.189-201, 1988.

GOLDSCHMIDT, E.E.; MONSELISE, S.P. Hormonal control of flowering in citrus and some other woody perennials. In: CARR, D. Plant growth substances, Berlin: Springer Verlag, 1970, p.758766.

GOLDSCHMIDT, E.E.; GOLOMB, A. The carbohydrate balance of alternate-bearing citrus trees and the significance of reserves for flowering and fruiting. Journal of American Society for Horticultural Science, Alexandria, v.107, n.2, p.206-208, 1982.

GOLDSCHMIDT,E.E.; ASCHKENAZI, N.; HERZANO, Y;; SCHAFFER, A.A.; MONSELISE, S.P. A role for carbohydrate levels in the control of flowering in citrus. Scientia Horticulturae, Amsterdam, v.26, n.2, p.159-166, 1985.

IEA-INSTITUTO DE ECONOMIAAGRÍCOLA. Área e produção dos principais produtos da agropecuária do Estado de São Paulo. São Paulo: IEA, 2005. Disponível em: <http://www.iea.sp.gov.br/out/ banco/menu.php>. Acesso em: 23 nov. 2005.

KRAJEWSKI, A.J.; RABE, E. Citrus flowering: a critical evaluation. Journal of Horticultural Science, Kent, v.70, n.3, p.357-374, 1995.

MONSELISE, S.P.; GOREN, R. The role of internal factors and exogenous control in flowering, peel growth, and abscission in citrus. HortScience, Alexandria, v.13, n.2, p.134-139, 1978.
MOSS, G.I.; MUIRHEAD, W.A. Climatic and tree factors relating to the yield of orange trees. I. Investigations on 'Washington Navel' and 'Late Valencia' cultivars. Horticultural Research, Edinburgh, v.11, n.1, p.3-17, 1971.

ORTOLANI, A.A.; PEDRO Jr., M.J.; ALFONSI, R.R. Agroclimatologia e o cultivo de citros. In: RODRIGUEZ, O.; VIÉGAS, F.; POMPEU Jr., J.; AMARO, A.A. Citricultura brasileira, Campinas: Fundação Cargill, 1991.p.153-195.

PEDRO Jr., M.J.; ORTOLANI, A.A.; RIGITANO, O.; ALFONSI, R.R.; PINTO, H.S.; BRUNINI, O. Estimativa de horas de frio abaixo de 7 e de $13^{\circ} \mathrm{C}$ para regionalização da fruticultura de clima temperado no Estado de São Paulo. Bragantia, Campinas, v.38, n.13, p.123130, 1979.

PEREIRA, A.R.; ANGELOCCI, L.R.; SENTELHAS, P.C. Agrometeorologia: fundamentos e aplicações práticas. Guaíba: Livraria e Editora Agropecuária, 2002. 478p.

REUTHER, W. Citrus. In: ALVIM, P.T.; KOZLOWSKI, T.T. Ecophysiology of tropical crops. London: Academic Press, 1977. p.409-439.

ROLIM, G.S.; SENTELHAS, P.C.; BARBIERI, V. Planilhas no ambiente Excel $^{\mathrm{TM}}$ para os cálculos de balanços hídricos: normal, seqüencial, de cultura e de produtividade real e potencial. Revista Brasileira de Agrometeorologia, Santa Maria, v.6, n.1, p.133-137, 1998.

SOUTHWICK, S.M.; DAVENPORT, T.L. Characterization of water stress and low temperature effects on flower induction in citrus. Plant Physiology, Rockville, v.81, n.1, p.26-29, 1986.

THORNTHWAITE, C.W.; MATHER, J.R. The water balance: publications in climatology. New Jersey: Drexel Institute of Technology, 1955. 104p.

TUBÉLIS, A. Clima: fator que afeta a produção e qualidade da laranja. Laranja, Cordeirópolis, v.16, n.2, p.179-211, 1995.

VALIENTE, J.I.; ALBRIGO, L.G. Flower bud induction of sweet orange trees [Citrus sinensis (L.) Osbeck]: effect of low temperatures, crop load, and bud age. Journal of American Society for Horticultural Science, Alexandria, v.129, n.2, p.158-164, 2004. 